\title{
Staphylococcal Enterotoxin C in Synovial Fluid of Patients With Rheumatoid Arthritis
}

\author{
Ramezan Ali Ataee ${ }^{1}$; Mohammad Hosein Ataee ${ }^{2}$; Gholam Hosein Alishiri ${ }^{3}$; Davoud \\ Esmaeili ${ }^{4,}$ \\ ${ }^{1}$ Department of Medical Microbiology, Faculty of Medicine, Baqiyatallah University of Medical Sciences, Tehran, IR Iran \\ ${ }_{2}^{2}$ Research Center of Microbial, Baqiyatallah University of Medical Sciences, Tehran, IR Iran \\ ${ }^{3}$ Department of Rheumatology, Baqiyatallah University of Medical Sciences, Tehran, IR Iran \\ ${ }^{4}$ Department of Medical Microbiology, Microbial Research Center, Baqiyatallah University of Medical Sciences, Tehran, IR Iran \\ ${ }^{*}$ Corresponding Author: Davoud Esmaeili, Department of Medical Microbiology, Microbial Research Center, Baqiyatallah University of Medical Sciences, P. O. Box: 19395-5487, Tehran, \\ IR Iran. Tel: +98-9124395884, E-mail: esm114@gmail.com
}

Received: November 9, 2013; Revised: December 7, 2013; Accepted: January 17, 2014

Background: In the previous studies using the commercial ELISA kit, the existence of staphylococcal superantigens has been reported in synovial fluid of patients with rheumatoid arthritis (RA).

Objectives: This study aimed to design molecular methods to detect staphylococcal enterotoxin C in synovial fluid of patients with rheumatoid arthritis.

Materials and Methods: In this experimental study, Staphylococcus aureus strain producing enterotoxin C was used as the reference strain. The polymerase chain reaction (PCR) was set up by design a specific pair of primers. Besides bacterial culture, 50 synovial fluid samples of patients with rheumatoid arthritis were subjected to DNA extraction, and then PCR amplification was carried out according to the protocol. All samples were examined by ELISA method for enterotoxin C. The data were descriptively analyzed.

Results: The results of bacterial culture were negative for all samples. The results showed that $66 \%$ (33 cases) of samples contained entC gene and only $46 \%$ (23 cases) have also enterotoxin C. The interesting finding was that the results of ELISA and PCR were the same and have shown only 22 positive cases (44\%samples) for staphylococcal enterotoxin C.

Conclusions: Based on the findings of this study, S. aureus enterotoxin C (SEC) has been detected in synovial fluid of patients with rheumatoid arthritis by PCR and ELISA methods. These valuable findings may describe the exact etiology of the RA and as well as change the methods of its diagnosis and treatment. This is the first research, which has shown the staphylococcal entC gene in synovial fluid of RA patients. However, S. aureus strains can produce more than 20 types of enterotoxins. Therefore, its involvement on rheumatoid arthritis pathogenesis makes an important challenge in the future. In this regard, further investigation on the other enterotoxins is necessary.

Keywords:Staphylococcal Enterotoxin C; Synovial Fluid; PCR, ELISA; Rheumatoid Arthritis

\section{Background}

The etiology of rheumatoid arthritis (RA) has still remained unclear and the disease is a substantial public health concern with its increasing costs, morbidity and disability. In this regard, many researches have been carried out. However, physiopathology of RA remains a mystery (1). The results of the researches showed that RA is an autoimmune disease in which the pathological immune reaction is initiated by the presentation of an antigen or superantigen by MHC class II positive cells to CD4 T cells (2). In this study, the role of superantigen in RA has been investigated by assaying the serum levels of staphylococcal enterotoxin B (SEB) antibodies and showed that RA patients had increased levels of serum IgM SEB antibody compared with normal subjects (3). However, Staphylococcus aureus can produce a great variety of toxins, with superantigen activity 3 .

Two classes of superantigens are toxic shock syndrome toxin 1 (TSST-1) and staphylococcal enterotoxins (SEs). Twenty types of SEs have been defined so far and the most common of them are as follows: SEA, SEB, SEC1, SEC2, SEC3, SED, and SEE (4). It is unclear whether they are involved in the pathogenesis of RA. One investigator has discussed the pathogenesis of arthritis induced by SEB, and this supports the possibility that superantigen may play a role in the induction of autoimmune diseases (5). In addition, current evidence suggests that the experimental arthritis model may provide a means to examine the role of superantigens and the efficacy of pharmacological agents for the treatment of RA.

Thus, the ability of enterotoxins as superantigen to activate enormous numbers of T cells suggests that they may play a major role in the course of autoimmune disorders (6). The results of Baillet research have shown that the concentrations of S100A8, S100A9, and S100A12 proteins

Copyright (C) 2014, Iranian Red Crescent Medical Journal; Published by Kowsar. This is an open-access article distributed under the terms of the Creative Commons Attribution-NonCommercial 4.0 International License (http://creativecommons.org/licenses/by-nc/4.0/) which permits copy and redistribute the material just in noncommercial usages, provided the original work is properly cited. 
are dramatically increased in synovial fluid of patients suffering from RA (7), but this research neglected the characteristics of these proteins. The results of another in vitro investigation indicate that activated synovial cells are potent antigen-presenting cells for SEB to T cells, suggesting that superantigens may play a critical role in the pathogenesis of RA through activating synovial cells (8). All researches conducted on the role of staphylococcal superantigens (enterotoxins) in the pathogenesis of RA were dependent on the results of cell culture, antistaphylococcal antibody measurements in serum, and injected staphylococcal enterotoxin B into the experimental mice. However, it is unclear whether the other staphylococcal enterotoxins have a role in the pathogenesis of RA. In fact, all the researches carried out in this regard were controversial. So far, no researches have directly shown staphylococcal enterotoxins in synovial fluid of patients.

\section{Objectives}

The purpose of this study was to track the Staphylococcus aureus enterotoxin $C$ in the synovial fluid of patients with rheumatoid arthritis.

\section{Materials and Methods}

\subsection{Bacterial Strain}

In this study, a strain of Staphylococcus aureus (S. aureus) with previously confirmed enterotoxin $C$ gene and its toxigenicity was used as the standard strain (8).

\subsection{Primer Design}

Using online Genscript software, a primer was designed on the basis of the reference sequence (S. aureus MW2 enterotoxin $C$ gene, accession number AB084256.1) and was analyzed by primer3 software. In addition, multiple alignments were carried out by DNASIS MAX trial version. This primer pair was amplified a 206-bp fragment.

\subsection{Sampling}

In an experimental study, during 18 months, from September 2010 to March 2012, a total of 50 samples of synovial fluid (SF) were provided by rheumatologist from patients with RA referred to clinical rheumatology. The collected SF samples were studied separately at the Microbiology Laboratory of Baqiyatallah University of Medical Sciences, Tehran, Iran. All patients participated in this study were briefed and taken informed consent. According to ACR (American College of Rheumatology) 2010 criteria (10), those who were diagnosed with RA, were included in the study. In aseptic conditions, each SF sample was separated into two parts; the first part was immediately inoculated in Castaneda medium. The other part was subjected to ELISA and PCR. The remaining samples of synovial fluid were kept at $20^{\circ} \mathrm{C}$ for the next stages of the research. This study was approved by Ethics Com- mittee of Baqiyatallah University of Medical Sciences (November 29, 2011, Code No: 24 paragraph 28).

\subsection{DNA Extraction}

DNA extraction of standard bacteria was performed by modified salting out method (11) from 24-hour culture suspension of bacteria. Then, the extracted DNA was evaluated by NanoDrop (Thermo Scientific NanoDrop 2000 Spectrophotometer USA) and conditions were optimized in order to perform PCR.

\subsection{DNA Amplification}

PCR was performed in optimum conditions for amplification of $206 \mathrm{bp}$ fragment in a total volume of $25 \mu \mathrm{L}$, which included $1 \mu \mathrm{L}(50 \mathrm{ng} / \mu \mathrm{L})$ of template DNA, $1.5 \mathrm{U}$ Taq DNA polymerase, $0.12 \mathrm{mM}$ dNTP Mix, $10 \mathrm{pmol}$ of each primer and $2 \mathrm{mM}$ MgCL2. DNA amplification was performed in a thermal cycler (Eppendorf AG 22331 Germany) using the following conditions: initial denaturation for $4 \mathrm{~min}$ at $94^{\circ} \mathrm{C}$ followed by 35 cycles of denaturation $\left(94^{\circ} \mathrm{C}\right.$ for $\left.30 \mathrm{~s}\right)$, annealing at $61^{\circ} \mathrm{C}$ for $30 \mathrm{~s}$ and extension at $72^{\circ} \mathrm{C}$ for $20 \mathrm{~s}$. The final extension step at $72^{\circ} \mathrm{C}$ for $5 \mathrm{~min}$ was performed after completing the cycles. As a positive control, PCR containing template DNA extracted from the standardized strain of Staphylococcus aureus.

\subsection{Visualization of Amplified DNA}

About $5 \mu \mathrm{l}$ aliquot of the PCR product was analyzed on 1.5\%TBE agarose (Maxpure agarose, Lot No. K452410. Spain). The electrophoresis was carried out in horizontal gel tanks at $100 \mathrm{mV}$ for $45 \mathrm{~min}$ or until the desired resolution was obtained. Then, agarose slab gel was stained with submerging in ethidium bromide solution $(0.5 \mathrm{mg} /$ $\mathrm{mL}$ ) for $10 \mathrm{~min}$ and after that washing was carried out. Gel were viewed by UV Transillumination (Bio-Rad, Universal Hood II, USA) and photographed using a $35 \mathrm{~mm}$ camera.

\subsection{DNA Extraction From Synovial Fluid}

DNA extraction from synovial fluid was performed according to kit instructions (CinnaPure DNA; Cat. No. PR881612; CinnaGen Co; Iran). Extracted DNA was examined by NanoDrop and $1 \mu \mathrm{L}$ of it was used as the template to perform PCR.

PCR product was electrophoresized by 1.5\% TBE agarose gel. Then, it was placed in ethidium bromide solution for $10 \mathrm{~min}$. After that, gel documentation was studied by ultraviolet light. Because additional bands were observed in the gel, the PCR product was electrophoresized again by $1.5 \%$ low melting temperature of agarose gel. Then, the equal band was isolated from the gel and DNA extraction was performed by the kit (Gel DNA Recovery Kit, GFGP-050, and Cinnagen, Iran). After ensuring of no other bands in the gel, it was sent to CinnaGen Co. to determine the sequence. 
Ataee RA et al.

\subsection{ELISA Plate Design}

For this purpose, antistaphylococcal enterotoxin C antibody (Rb PAb enterotoxin C, Ab 15899, 500 Ug, Lot \#722163; Abcam Germany) was used. The antibody was a monospecific polyclonal one. Bovine Serum albumin (Lot\#057K0737, Sigma Life Science), horseradish peroxidase conjugated Ab (Anti-Rabbit IgG, Code No: AP7181, Lot\#102, Razi BioTech, Iran), substrate (urea peroxide) and Cologne (tetramethylbenzidine) and stopper (sulfuric acid) were purchased from Merck, Germany. Primary antibody (Staphylococcus aureus enterotoxin C antibodies) was coated onto the plates. The working dilution was $1 \mathrm{ng} / \mu \mathrm{L}$. Each one of 96-well microplate ELISA was selected. In each plate, the A, B, C, D, and E rows were coated with anti-enterotoxin type $\mathrm{C}$ antibody. Rows $\mathrm{F}$ and $\mathrm{G}$ were considered as negative controls. Row $\mathrm{H}$ was selected as a positive control. In negative control rows, the antibody was not coated. For positive control row, a specific antibody of a known toxin was used. The plate prepared by the above mentioned method was put at $37^{\circ} \mathrm{C}$ overnight so the remaining liquid evaporated. Then, all wells were treated with 3\% BSA solution. This was done in order to saturate free binding sites. After that, plates were sailings in aluminum foil pocket and stored in a refrigerator at $4^{\circ} \mathrm{C}$ until further use.

After preparation of ELISA plates, all samples were assayed as follows: $50 \mu \mathrm{L}$ of synovial fluids (diluted with sterile PBS to 50\%) was loaded in each well and the plates were incubated $a t 37^{\circ} \mathrm{C}$ for one hour.After theincubation period, the remaining samples were evacuated from the plates, and they were washed three times by washing buffer for $45 \mathrm{~s}$. After that, $50 \mu \mathrm{L}$ of working diluted the secondary antibody was added to each well. Next, they incubated for $50 \mathrm{~min}$ at $37^{\circ} \mathrm{C}$ and then the sink was drained and washed three times. In the final stage, $50 \mu \mathrm{L}$ of substrate solution (urea peroxide) and then $50 \mu \mathrm{L}$ chromo gene solution (tetramethylbenzidine) was added. After 15 minutes and checking the color, the plates were subjected to $100 \mu \mathrm{L}$ of stopper (sulfuric acid $2 \mathrm{M}$ ) and immediately poured into each well. Then, the opacity of each well of ELISA plates at $450 \mathrm{~nm}$ were measured using ELISA Reader (Tecan, Austia Gmbh, Model: Sunrise, Serial No: 501000095). The cut off value was calculated using the mean OD of negative controls, and a constant factor of 0.15 was added to them, till the test cut-off value was obtained.

\section{Results}

The results of demographic analysis showed that 35 patients were male and 15 of them were female. Their average age was $49 \pm 12 \mathrm{y}$. None of the patients was taking medication. In addition, there were no histories of the surgery, hepatitis, jaundice, and hijama (phlebotomy). The results of bacteriological culture of 50 samples of synovial fluid from patients with rheumatoid arthritis were reported negative. In other words, despite the use of enriched bacteriologic culture media, bacteria grew in none of the samples.

\subsection{The Results of ELISA Test}

The designed ELISA plate could reveal the presence of enterotoxin C of Staphylococcus aureus in some samples. In fact, the results of ELISA test on synovial fluid samples showed the presence of enterotoxin C in 22 cases (44\%). Based on cut-off value and also positive control wells, the C row in Figure 1 has shown that 17, 18, 22 and 24 wells are positive samples. The $\mathrm{H}$ row is positive control with 2-10 ng toxin.

As it can be seen in Figure 1, enterotoxin type C of Staphylococcus aureus is shown in row $\mathrm{C}$ of the samples 17, 22, 24 and 25 . In this plate, rows $F$ and $G$ are negative controls (data not shown) and row $\mathrm{H}$ is a positive control with different concentrations of standard value.

Positive or negative ELISA test criteria were calculated by cut-off point calculation so that the constant coefficient 0.15 was added to the average absorption rate of the negative control wells (wells in row F and G); the resulting number was cut-off point. Wells that have absorption rate higher than cut-off point are considered positive and the wells that have less absorption rate than cut-off point are considered negative.

Measurement of absorption rate in an ELISA plate that was designed to detect staphylococcal enterotoxins with a wavelength of $450 \mathrm{~nm}$ and the cut-off value of this ELISA plate is obtained by mean calculation of absorption rate of negative control wells and adding a fixed number (0.15) to it. Thus the mean absorption of the negative control wells has been achieved at 0.129 and if the fixed number (0.15) is added to it, the number 0.279 will be obtained. Therefore, the wells that have absorption rate higher than 0.279 are considered positive and the wells which have less absorption rate than 0.279 are considered negative samples.

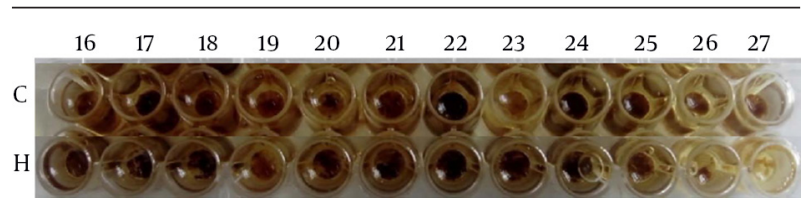

Figure 1. An Example of ELISA plate Designed in This Study

\subsection{The Results of Genome Extraction From Sam- ples of Synovial Fluid}

The results of genome extraction from samples of synovial fluid of patients with RA and their electrophoresis suggested that the DNA quantity was different in samples (Figure 2). The measurement of the extracted DNA concentration showed the difference by NanoDrop. As the maximum amount was related to sample 23 with $127 \mathrm{ng} / \mu \mathrm{L}$, and the minimum amount was related to sample 25 with $12 \mathrm{ng} /$ $\mu \mathrm{L}$. As shown in Figure 2, loading $1 \mu \mathrm{L}$ of sample 25 was not associated with a distinct band. 
The results of primer designing were as follows; F- GAAACACAATTTCTTTTGAAGTGC and R- AAACTTATCGCCTGGTGCAG which were amplified at 206-bp amplicons as PCR products. The results of samples PCR with amplifier primer of a 206-bp fragment suggested that in 26 out of 50 samples, there was a part of entC gene. Figure 3 shows the result of PCR product electrophoresis with amplifier primer of a 206-bp fragment for a number of samples.

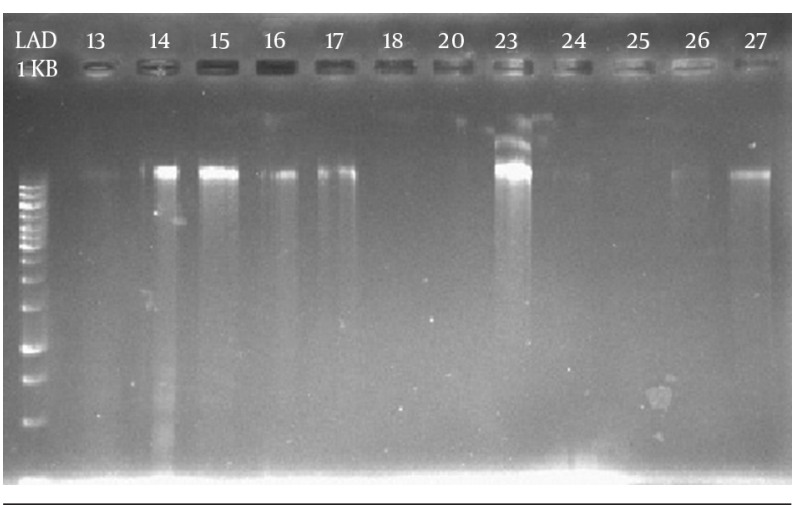

Figure 2. The Results of Electrophoresis of Extracted DNA of a Number of Synovial Fluid Samples

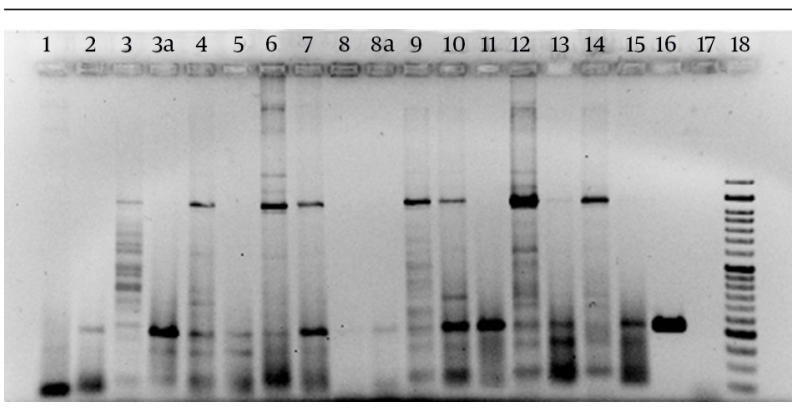

Figure 3. The Result of PCR Product Electrophoresis with Amplifier Primer of a 206-bp Fragment

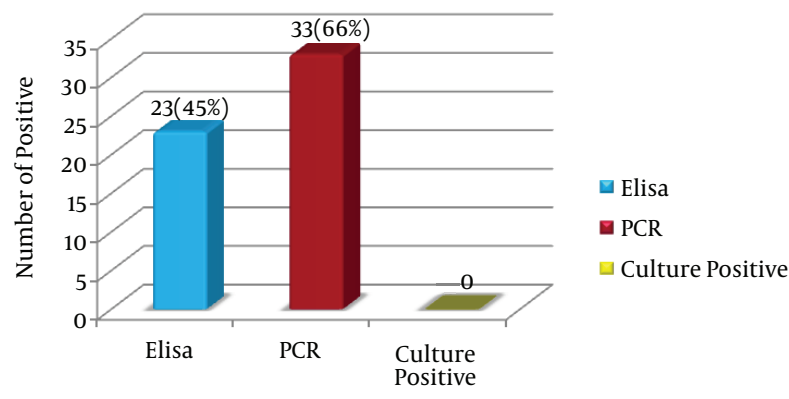

Name of Tests

Figure 4. Comparison of PCR and Elisa Results are Shown

Look at the positive control bands in line 16 and the bands with the same width in samples 2, 3a, 4, 5, 6, 7, 8a, 9, 10, 11, 12 , and 15 lines. Lines 16 and 17 in positive and negative con- trol, respectively and line 18 are 50-bp molecular markers. The results of PCR method indicated that 33 (66\%) out of 50 synovial fluid samples (100\%) of patients with rheumatoid arthritis contained the enterotoxin $C$ gene (Figure 4). Sequencing of PCR products of one sample as representative of other samples showed 99\% similarity with the reference gene for staphylococcal enterotoxin C. While the results of ELISA test showed that only 23 cases (46\%) have enterotoxin C. The comparison of PCR and ELIZA results revealed that, they were not $100 \%$ matched. In 11 cases (22\%) of samples tested by PCR, enterotoxin C gene is shown while the negative results were obtained for these samples by ELISA. Similarly, the results of ELISA showed the presence of enterotoxin $C$ in 2 cases (4\%), whereas a negative PCR result was reported for those cases. In fact, only 22 cases (44\%samples) between PCR and ELISA have shown similarity. Analysis results revealed that in 10 cases (20\%) neither PCR method, nor ELISA showed entC gene or enterotoxin $C$ protein.

\section{Discussion}

In this study, the enterotoxin type $C$ existence in synovial fluid of patients with RA was demonstrated by ELISA and PCR methods and their confirmation with sequencing. However, there is a report which has claimed the lack of evidence for the role of staphylococcal enterotoxins in RA (12).

The ELISA technique could show the presence of large amounts of enterotoxin C in 23 samples (46\%) out of 50 samples of synovial fluid. Also, the results of using PCR molecular method and amplifier primer of a 206-bp fragment showed the presence of 206-bp fragment, which is enterotoxin $C$ gene in 33 samples (66\%) out of 50 samples. Our previous study revealed that in some cases, more than one enterotoxin was detected in synovial fluid samples (13). It can be assumed that a similarity between enterotoxins has led to this difference. In this study, the specific antibody used has only detected enterotoxin type C. But, do Staphylococcus aureus enterotoxins such as enterotoxin $C$ really play a role in the pathogenesis of rheumatoid arthritis? It is not clear. To answer to this question, further studies are needed. No report has been documented so far regarding the presence of staphylococcal enterotoxins in the synovial fluid of patients with rheumatoid arthritis. This study is the first one which demonstrated proteins in the synovial fluid of patients with rheumatoid arthritis that react to antistaphylococcal enterotoxins $\mathrm{C}$ antibody. One of the most important evidence for this fact is PCR technique with specific primers, which can amplify 206 amplicon and its results were similar to positive control. In fact, this study has answered to an important question.

The main finding of this study was that, in addition to the presence of a protein with properties of staphylococcal enterotoxin, there is a sequence of enterotoxin type $\mathrm{C}$ coding genes. It should be noted that enterotoxin type $\mathrm{C}$ of Staphylococcus aureus is one of the superantigenic 
enterotoxins with three subtypes (C1, C2 and C3) (14). The result of sequencing indicated that the amplified gene in synovial fluid of patients with rheumatoid arthritis is enterotoxin C1 gene. Enterotoxin C subtypes have shown different biological effects. One of the most important biological effects of enterotoxin C subtypes of Staphylococcus aureus is their progeny effects (15). Furthermore, the enterotoxins like enterotoxin type B stimulates production of different cytokines by combining with MHC (major histocompatibility complex) class II receptors and T cells stimulation (16). It also as a superantigen deeply affects the immune system. The results of a survey showed that $70 \%$ of MRSA (Methicillin-resistant Staphylococcus aureus) strains isolated from patients' blood were able to produce TSST-1 and enterotoxin C and the remaining 30\% have produced other enterotoxins (17).

Despite treating staphylococcal infection, these patients were diagnosed with arthritis and eczema. These finding may confirm that superantigenic enterotoxins are involved in causing diseases. Furthermore, in patients with nasal polyps, the presence of IgE of antienterotoxins A and B of staphylococci has been shown (18). Although a comprehensive study in these fields is will debate with available, the results of the existing researches indicate that superantigenic enterotoxins are effective in the pathogenesis of chronic inflammatory diseases. However, it has been shown that enterotoxin $C$ has been isolated by different strains of Staphylococcus aureus, or their products such as milk and its by-products (19). Of course, detection of enterotoxin $\mathrm{C}$ in different samples is required, because food poisoning outbreak caused by staphylococcal enterotoxins has produced some concerns and shown the lack of validation of some pasteurization systems (20). Moreover, toxigenicity of clinical strains of Staphylococcus aureus was investigated, and it was found that $61.3 \%$ of strains isolated from patients produced enterotoxin C (21).

The important result of the above-mentioned study is that some of the Staphylococcus aureus strains have produced more than one enterotoxin. A similar result was found in the present study. Furthermore, enterotoxin production has been reported from coagulase-negative strains isolated from livestock (22) or strains isolated from patients (23). However, designing systems for rapid detection of food contaminations or toxigenicity of isolated bacterial strains have attracted scientists' attention. For example, in 2002, immunoassay system was introduced for detection of enterotoxin $C$ in food samples (24). Given the above-mentioned cases, in this study, a test with high sensitivity, high specificity and repeatability was designed and used that included ELISA method as well as PCR molecular method for detection of staphylococcal enterotoxin type $C$ and 50 samples of synovial fluid of patients with rheumatoid arthritis were studied.

In this study, by using an enterotoxin $C$ producing strain, PCR molecular method was first optimized for detection of the gene coding of staphylococcal enterotoxin
C. Then, the results obtained from PCR were compared with sandwich ELISA method. The results indicated that $66 \%$ (33 cases) of samples contained entC gene and only $46 \%$ ( 23 cases) have also enterotoxin C. The main finding was that the results of ELISA and PCR were the same and have shown only 22 positive cases ( $44 \%$ samples) for enterotoxin C. In addition, this method showed high sensitivity and specificity for detection of enterotoxin C.

The strong points of this study are its findings that may partially explain the etiology of RA as well as change the methods of diagnosis and treatment of the RA disease. This is the first research that has shown the staphylococcal entC gene in synovial fluid of RA patients. The weak points of this study relate to this fact the S. aureus strains can produce more than 20 types of enterotoxins (classical superantigen). Thus, involvement of them in rheumatoid arthritis pathogenesis made an important challenge for future studies. In this regard, further investigation on the other enterotoxins is necessary.

\section{Acknowledgements}

The authors would like to thank the head of Faculty of Medicine, Dr. Jamal Akhavan, for encouraging the authors.

\section{Authors' Contributions}

Ramezan Ali Ataee designed and developed the original idea. Gholam Hosein Alishiri prepared the synovial fluid samples. Ramezan Ali Ataee, Davoud Esmaeili, and Mohammad Hosein Ataee had performed the experimental protocol and abstracted and wrote the manuscript.

\section{References}

1. Seror R, Pagnoux C, Guillevin L. Plasma exchange for rheumatoid arthritis. Transfus Apher Sci. 2007;36(2):195-9.

2. Zangerle PF, De Groote D, Lopez M, Meuleman RJ, Vrindts Y, Fauchet F, et al. Direct stimulation of cytokines (IL-1 beta, TNFalpha, IL-6, IL-2, IFN-gamma and GM-CSF) in whole blood: II. Application to rheumatoid arthritis and osteoarthritis. Cytokine. 1992;4(6):568-75.

3. Origuchi T, Eguchi K, Kawabe Y, Yamashita I, Mizokami A, Ida H, et al. Increased levels of serum IgM antibody to staphylococcal enterotoxin B in patients with rheumatoid arthritis. Ann Rheum Dis. 1995;54(9):713-20.

4. Pinchuk IV, Beswick EJ, Reyes VE. Staphylococcal enterotoxins. Toxins (Basel). 2010;2(8):2177-97.

5. Omata S, Sasaki T, Kakimoto K, Yamashita U. Staphylococcal enterotoxin B induces arthritis in female DBA/ 1 mice but fails to induce activation of type II collagen-reactive lymphocytes. Cell Immunol.1997;179(2):138-45.

6. Nagai H, Takaoka Y, Kamada H, Mori H. The model of arthritis induced by superantigen in mice. Life Sci.1994;55(12):PL233-7.

7. Baillet A. [S100A8, S100A9 and S100A12 proteins in rheumatoid arthritis]. Rev Med Interne. 2010;31(6):458-61.

8. Origuchi T, Eguchi K, Kawabe Y, Mizokami A, Ida H, Nagataki S Synovial cells are potent antigen-presenting cells for superantigen, staphylococcal enterotoxin B (SEB). Clin Exp Immunol. 1995; 99(3):345-51.

9. Ataee RA, Kamali M, Karami A, and Ghorbani M. Standardization of molecular method detection of the entC in Staphylococcus aureus isolated from human infections and determine it's sequence. Iran J Mil Med. 2012; 14(3): 232-40. 
10. Aletaha D, Neogi T, Silman AJ, Funovits J, Felson DT, Bingham C3, et al. 2010 Rheumatoid arthritis classification criteria: an American College of Rheumatology/European League Against Rheumatism collaborative initiative. Arthritis Rheum. 2010; 62(9):2569-81.

11. Sambrook J, Russel DW. Molecular Cloning: a laboratory manual. 3 edNew York: Coldspring Harbour Laboratory Press; 2001. pp. 417-21.

12. Liu MF, Li JS, Lin YS, Lei HY. Lack of evidence for the role of staphy lococcal enterotoxins in rheumatoid arthritis. Clin Exp Rheumatol.1997;15(1):67-70.

13. Ataee RA, Alishiri GH, Mohoseni- Moghadam Z, Latifi AM, Ataee MH. Enzyme-linked Immunosorbent Assay for Detection Staphylococcal Enterotoxins in Synovial fluid of heumatoid Arthritis patients. Pur Applied Microbiol J. 2013;7(2):1113-9.

14. Reiser RF, Robbins RN, Noleto AL, Khoe GP, Bergdoll MS. Identification, purification, and some physicochemical properties of staphylococcal enterotoxin C3. Infect Immun. 1984;45(3):625-30.

15. Su CL, Cheng CC, Lin MT, Yeh HC, Lee MC, Lee JC, et al. Staphylococ cal enterotoxin C1-induced pyrogenic cytokine production in human peripheral blood mononuclear cells is mediated by NADPH oxidase and nuclear factor-kappa B. FEBS J. 2007;274(14):3633-45.

16. Hui J, Cao Y, Xiao F, Zhang J, Li H, Hu F. Staphylococcus aureus enterotoxin C2 mutants: biological activity assay in vitro. J Ind Microbiol Biotechnol. 2008;35(9):975-80.

17. Mizukane R, Nakatomi M, Futsuki Y, Araki J, Asai S, Sawatari K, et al. [A study of virulence factors produced by MRSA strains isolated from blood samples]. Kansenshogaku Zasshi.1998;72(8):788-93.

18. Fan YP, Xu G, Zuo KJ, Xu R, Jiang HY, Lin ZB, et al. [Detection of specific IgE of anti-Staphylococcus aureus enterotoxins in nasal polyps and analysis theoretically about the superantigen hypothesis]. Zhonghua Er Bi Yan Hou Tou Jing Wai Ke Za Zhi. 2006;41(11):825-9.

19. Podkowik M, Park JY, Seo KS, Bystron J, Bania J. Enterotoxigenic potential of coagulase-negative staphylococci. Int J Food Microbiol.2013;163(1):34-40.

20. Weiler N, Leotta GA, Zarate MN, Manfredi E, Alvarez ME, Rivas M [Foodborne outbreak associated with consumption of ultrapasteurized milk in the Republic of Paraguay]. Rev Argent Microbiol. 2011;43(1):33-6.

21. Cao H, Wang M, Zheng R, Li X, Wang F, Jiang Y, et al. [Investigation of enterotoxin gene in clinical isolates of Staphylococcus aureus]. Nan Fang Yi Ke Da Xue Xue Bao. 2012;32(5):738-41.

22. de Freitas Guimaraes F, Nobrega DB, Richini-Pereira VB, Marson PM, de Figueiredo Pantoja JC, Langoni H. Enterotoxin genes in coagulase-negative and coagulase-positive staphylococci isolated from bovine milk. J Dairy Sci. 2013;96(5):2866-72.

23. Ataee RA, Mehrabi-Tavana A, Izadi M, Hosseini SM, Ataee MH. Bac terial meningitis: a new risk factor.JRes Med Sci. 2011;16(2):207-10.

24. Fluer FS, Prokhorov V, Vesnina AF, Akatov AK. [Enzyme immunoassay system for detection of staphylococcal enterotoxin, type C].Zh Mikrobiol Epidemiol Immunobiol. 2002(6):65-8. 